\title{
11a. Die Profundaplastik in der Behandlung arterieller Verschlußerkrankungen im Stadium IV
}

\section{SPERLTNG und H. SchotT (a.G.)}

Chirurgische Universitätsklinik und -Poliklinik, Würzburg

Das Stadium IV der arteriellen Verschlußerkrankungen ist mit einer hohen Amputationsrate belastet. Periphere Nekrose oder Gangrän sind nicht selten Folge von Gefäßverschlüssen, welche sich über die Arteria femoralis communis und superficialis erstrecken. Auch die Arteria ilica externa kann in die Totalobliteration miteinbezogen werden. Meist ist bei dieser Verschlußkombination die distale Obliteration im Femoralis superficialis-Abschnitt einer operativen Korrektur aus lokalen Gründen wegen eingeschränkter Abflußverhältnisse nicht zugänglich. Diesen Patienten droht die hohe Amputation. In geeigneten Fällen gelingt durch Wiedereröffnung des Profundakreislaufes die Erhaltung der Extremität, die Abheilung von Nekrose oder Gangrän und die Rückführung der Verschlußerkrankung in das Stadium II.

Die Profundaplastik stellt einen risikoarmen Eingriff dar, der auch in höherem Alter gut toleriert wird. Die Operation kann gegebenenfalls auch in Lokalanaesthesie durchgeführt werden. Auf eine ausgedehnte Revision der Beckenarterien darf bewußt verzichtet werden. Entscheidend ist die Erzielung eines ausreichenden Einstromes in die Arteria femoralis profunda.

An operativen Verfahren stellt die offene Desobliteration der Femoralisgabel mit Ausschälung der Anfangsstrecke der Arteria femoralis profunda die Methode der Wahl dar. Arteria femoralis communis und Arteria ilica externa lassen sich von der gleichen Arteriotomie retrograd mit einem Ringstripper desobliterieren. Die Erweiterung der Arteriotomie mit einem venösen Streifentransplantat ist bei geringem Lumen der Arteria femoralis communis und einer bis in die Arteria femoralis profunda geführten Arteriotomie erforderlich.

Durch eine entsprechende Bildserie werden die Ergebnisse der Profundaplastik aufgezeigt.

\section{1b. Intraoperative Farbdarstellung der Gallenwege bei der kongenitalen Gallengangsatresie}

\section{G. BÖTTGER}

Chirurgische Universitätsklinik Würzburg

(Direktor: Prof. Dr. E. Kern)

Die operative Darstellung der extrahepatischen Gallenwege bei der kongenitalen Gallengangsatresie erfordert nicht selten ein präparatori- 